\title{
Gestión del conocimiento como herramienta para la gestión administrativa en el sector Guasmo Sur - Guayaquil
}

\section{Knowledge management as a tool for the administrative management in the South Guasmo - Guayaquil}

Evelyn Verónica Madrid-Mosquera

Universidad de Guayaquil, Guayaquil, Ecuador

evelyn.madridm@ug.edu.ec

https://orcid.org/0000-0001-9851-4605

Maribel Alexandra Clemente-González

Universidad de Guayaquil, Guayaquil, Ecuador

maribel.clementeg@ug.edu.ec

(iD https://orcid.org/0000-0002-2272-2210

Jhoanne Danniela Infante-Jaramillo

Universidad de Guayaquil, Guayaquil, Ecuador

daniela.infantej@ug.edu.ec

(D) https://orcid.org/0000-0001-9542-3185

Estrella Zulema Rivera-Aguilar

Universidad de Guayaquil, Guayaquil, Ecuador

estrella.riveraa@ug.edu.ec

(D) https://orcid.org/0000-0001-8891-3629

Recepción: 24/07/2021 | Aceptación: 19/10/2021 | Publicación: 30/10/2021

Cómo citar (APA, séptima edición): Madrid-Mosquera, E. V., Clemente-González, M.A., Infante-Jaramillo, J.D., y Rivera-Aguilar, E. Z. (2021). Gestión del conocimiento como herramienta para la gestión administrativa en el sector Guasmo Sur - Guayaquil. INNOVA Research Journal, 6(3.1), 19-40. https://doi.org/10.33890/innova.v6.n3.1.2021.1847

\section{Resumen}

Las tiendas de barrios son fuentes de ingresos económicos para las familias que, por falta de aplicación de conocimientos administrativos, impiden que se adapten a sus necesidades, frente a la 
competencia y por ende puedan provocar el cierre de estos. La importancia del presente estudio reside en dar a conocer opciones para mejorar las dificultades administrativas que atraviesan en sus actividades cotidianas. Factores como el tiempo, dinero, el desconocimiento entre otros, son considerados como parte de las causas que impiden el aprendizaje y crecimiento de estos. Por lo que, para contrarrestar la problemática existente, se estableció como objetivo general realizar un análisis situacional para poder conocer las gestiones administrativas que manejan los micronegocios denominados tiendas de barrio del Guasmo Sur en la ciudad de Guayaquil. La metodología de investigación que se empleó es mixta, donde la recolección de datos se realizó por medio de las herramientas como encuesta, entrevistas y observaciones dirigidos a los dueños y clientes de tiendas pertenecientes a la localidad del Guasmo Sur de nuestra ciudad porteña. Por lo cual concluimos, que las tiendas de barrio del Guasmo Sur, en su gran mayoría son administrados por miembros de una misma familia, siendo el $45,9 \%$ de estos negocios la única fuente de ingresos. Estos micronegocios a su vez asumen conductas empresariales en menores escalas, por lo que podrían mejorar la administración del negocio y la atención al cliente al conocer la importancia de la gestión administrativa.

Palabras claves: tiendas de barrio; gestión del conocimiento; estrategias; Guasmo Sur.

\begin{abstract}
Neighborhood stores are a source of economic income for families that, due to lack of application of administrative knowledge, prevent them from adapting to their needs, in the face of competition and therefore can cause their closure. The importance of this study lies in making known options to improve the administrative difficulties that they go through in their daily activities. Factors such as time, money, ignorance among others, are considered as part of the causes that impede their learning and growth. Therefore, to counteract the existing problem, a general objective was established to carry out a situational analysis to be able to know the administrative procedures handled by the micro-businesses called Guasmo Sur neighborhood stores in the city of Guayaquil. The research methodology used is mixed, where the data collection was carried out through tools such as surveys, interviews and observations directed at the owners and customers of stores belonging to the town of Guasmo Sur in our Buenos Aires city. Therefore, we conclude that the Guasmo Sur neighborhood stores are mostly managed by members of the same family, with $45.9 \%$ of these businesses being the only source of income. These micro-businesses, in turn, assume business behaviors on a smaller scale, so they could improve business administration and customer service by knowing the importance of administrative management.
\end{abstract}

Keywords: neighborhood stores; knowledge management; strategies; South Guasmo.

\title{
Introducción
}

En la ciudad de Guayaquil, el disponer de una tienda de barrio en diferentes sectores, tanto en zonas urbanas y vulnerables, es considerado como parte esencial de la cultura emprendedora que poseen los habitantes de esta ciudad, mostrándonos así la relación directa que existe por parte de las tiendas barriales y el papel que estos juegan dentro de la economía local y nacional. En la legislación ecuatoriana se puede encontrar información referente a la Pequeña y Mediana Empresa MiPymes, en el art. 53 del Código Orgánico de la Producción, Comercio e Inversiones COPCI 
(2010), el cual indica que la micro, pequeña y mediana empresa es toda persona natural o jurídica que, como una unidad productiva, ejerce una actividad de producción, comercio y/o servicios, y que cumple con el número de trabajadores y valor bruto de las ventas anuales, señalados para cada categoría, de conformidad con los rangos que se establecerán en el reglamento del mismo Código.

Tal como lo señala el COPCI, para considerar a un negocio de tiendas de barrio como micronegocios nos basamos en la definición de que esta debe ajustarse a características específicas, siendo esta la categoría que mejor define a las tiendas de barrio, con aspectos de informalidad, en la mayoría de los casos debido a la forma en que inician sus actividades comerciales bajo la figura de personas naturales en la parte legal además de la escasez de los recursos tanto humano, material y tecnológico que cuentan a disposición de los mismos. Dado el propósito de estudio de este trabajo, los términos; microempresas, micronegocios, negocios informales, sector informal, unidades de economía informal serán tomados en cuenta como sinónimos.

El sector informal es parte del conjunto de agentes productivos que escapan de los requisitos y las obligaciones que el Estado impone, en un ambiente legal donde actividades similares sí se encuentran reguladas. Estas actividades son excluidas (al menos parcialmente) de los beneficios que el estatus de formalidad les otorga (Portes y Benton, 1987). Por tanto, en esta definición pueden ser incluidas actividades provenientes del comercio, servicios, manufactura entre otros. Con las particularidades indicadas previamente, procedemos a estudiar los diversos aspectos que se presentan en las tiendas de barrio, además de la participación que estas tienen al ser consideradas como agentes en actividades productivas dentro de la economía guayaquileña y ecuatoriana, lo cual nos permitirá conocer y entender la realidad que envuelve a estos negocios, como tal, al ser parte del aparato productivo, en términos generales.

Según datos del INEC (Instituto Nacional de Estadísticas y Censos), existieron alrededor de 15.223 tiendas de barrio en Guayaquil, estimando que el modelo de negocio generó empleo a más de 30 mil personas en aquel entonces, lo cual representó el 1,99\% del PEA (Población Económicamente Activa) de Guayaquil y produjo un volumen de ventas de más de \$531 millones en la urbe, de los \$56.603 millones del PIB (Producto Interno Bruto) en el año 2010.

Todas las argumentaciones describen de forma preliminar como antecede la necesidad en destacar que los micronegocios, en específico "tiendas de barrio" muestran un papel vital en la comercialización de productos de la economía local y nacional. Por esas razones estos negocios se encuentran presentes debido a las altas tasas de desempleo, ya que representan un soporte para la economía del hogar, en ese contexto de acuerdo con los resultados reflejados en el censo económico elaborado por el INEC (2010) en Ecuador constan cerca de 85.524 sociedades entre tiendas barriales, supermercados y locales con venta de suministros de alimentos. Por consiguiente, forman una importante fuente de ingreso a los miles de hogares que se han inclinado por acoger este modelo de negocio.

De acuerdo con el estudio de La Vitrina (2019) en el proyecto de investigación para tenderos de Bogotá detalla cuáles son las desventajas que presentan las tiendas de barrio, como lo es la contabilidad de método empírico, el aspecto financiero de muchos tenderos es que no lo saben manejar, por el temor de pasar del régimen simplificado al común. Los medios de pago son en base al uso del efectivo como único medio de pago o transacción no hay descuento en los productos, la 
parte tecnológica presenta la falta de elementos de control tecnológico pese a que está disponible, el propietario debe tener predisposición para capacitarse y usar los equipos y herramientas.

Villanueva (2018) periodista del diario El Universo menciona en una entrevista dirigido al director de la Red Ecuatoriana de Tenderos, quien afirmó que en Guayaquil existían alrededor de 16.000 tiendas, de acuerdo con un estudio de mercado que fue realizado por esta asociación en dicho año. Es así que, con las cifras citada, se pueden observar los diferentes escenarios que enfrentan los dueños de las tiendas del barrio del Guasmo Sur, al encontrarse establecidos en zonas vulnerables de la ciudad porteña, es por ello que se procedió a consultar a los propietarios de tiendas barriales de dicho sector, sobre los factores que podrían estar causando impedimentos en la adquisición de conocimientos que les permitan realizar un manejo más adecuado de los mismos, de manera eficiente.

\section{Tabla 1}

Delimitación de la investigación

\section{Delimitación}

\begin{tabular}{ll} 
Población: & Tiendas de barrio del Guasmo Sur en la ciudad de Guayaquil \\
Tiempo: & $2020-2021$ \\
Espacio: & Etapas del sector del Guasmo Sur de la ciudad de Guayaquil \\
\hline
\end{tabular}

Nota: Elaborado por Autoras

El objetivo general del presente trabajo de investigación es un diagnóstico situacional sobre las tiendas de barrio, por medio de instrumentos de recopilación de datos, para determinar el estado actual por el que atraviesan estos micronegocios.

\section{Marco Teórico}

En esta sección se analizan las teorías, conceptualizaciones y antecedentes que se consideren válidos para el desarrollo de la investigación. Hidalgo (2017) afirmó lo siguiente en una entrevista ofrecida al diario EL Telégrafo: "El Guasmo ha sido por mucho tiempo un punto de referencia en actividades comerciales informales, con presencia notoria de micro emprendedores de la misma comunidad."

Para La Vitrina (2019) se puede definir que la tienda de barrio "Es una unidad económica vital pues de ella dependen numerosas personas" (p. 7), las tiendas de barrio son el lugar donde las personas interactúan y realizan sus compras, con algunas prácticas muy comunes en su dinámica diaria como son las compras al fio, dada por la relación de confianza a nivel social, que existe con las personas propietarias de las tiendas.

Según La Vitrina (2019): "El tendero es una parte vital de la comunidad, por llevar a muchas familias los productos del consumo diario". (p. 7), un tendero pude ser tanto el dueño, el personal o un familiar encargado de la administración de los recursos de la tienda. 


\section{Figura 1}

Tipos de tiendas

\section{Por apariencia}

•Tienda pequeña, ordenada, aseada

\section{Por su familiaridad}

- Tienda familiar,popular, placenter, servicial, sacadora de apuros

\section{Por su rol de intermediación}

- Tienda mercadera, surtida, básica, todera, miniaturizadora

Nota: Elaborado por Autoras

Según Páramo (2015cómo se citó en Londoño Toro, 2018) menciona que el Grupo de Investigación en Marketing, conocida por sus siglas (GIM) de la Universidad del Norte, realizó una minuciosa investigación cualitativa en seis ciudades de Colombia entre las que detectaron 13 tipos de tiendas de barrio agrupadas en 3 categorías de acuerdo con apreciaciones culturales y utilidad que palpan los consumidores las cuales son: tiendas por su apariencia, por su familiaridad y por su rol de intermediación. Estas tiendas a su vez contienen otras subclasificaciones empezamos con el tipo de tiendas "por su apariencia", en donde una parte de los consumidores, observan en las tiendas un espacio físico características de tamaño, orden, distribución y limpieza, determinantes para mantener y alimentar sus relaciones permanentes Páramo (2015cómo se citó en Londoño Toro, 2018). Siendo así una tienda por su apariencia se puede categorizar como pequeña la cual define el tipo de negocio cómo en el interior de unos pocos metros cuadrados, donde el tendero exhibe sustanciales cantidades de productos en diferentes presentaciones, sobre todo las más pequeñas.

En el segundo grupo "tiendas por su familiaridad", consideradas, por las personas como algo cercano a sus propios mundos, siendo un espacio en el que el cliente se siente como estar en su propio hogar Páramo (2015 cómo se citó en Londoño Toro, 2018). Subclasificándose en tienda familiar, la cual tiene un doble carácter; ya que, por un lado, es un espacio que a pesar de la inseguridad en que vivimos, es visitado sin temores ni angustias por el ama de casa que se aprovisiona de los productos que requiere diariamente e inclusive por el menor de sus hijos, que hace mandados o busca un confite o una golosina. Esta tienda también es vista como un negocio de carácter estrictamente familiar. Es una organización en la que participan varios miembros de una misma familia, donde a pesar de las funciones laborales se siguen conservando las mismas relaciones de subordinación familiar. Y como último prototipo de tienda "por su rol de intermediación" son aquellas que cumplen el servicio vínculo logístico entre distribuidor y consumidor final Páramo (2015 cómo se citó en Londoño Toro, 2018) la cual puede subdividirse 
en una tienda mercadera por ser un espacio comercial y social donde los consumidores se aprovisionan de manera permanente. Es el sitio para hacer el mercado que requiere una familia, donde diaria, semanal o quincenalmente se hacen todas las compras.

\section{Gestión del Conocimiento}

La Gestión de Conocimiento se desarrolla en el marco de la llamada "nueva economía" o "Economía del conocimiento", en la que académicos y empresarios reconocen la importancia del conocimiento para lograr y mantener la ventaja competitiva de las organizaciones (Giraldo, 2018).

Es decir que la gestión del conocimiento siembra el intercambio de ideas, conocimiento y dialogo de saberes, por lo que este no debería ser un modelo rígido, cuando es dirigido a organizaciones informales, se debe tomar en consideración la experiencia y conocimientos naturales que posean los sectores en donde será aplicado dicho proceso, este tipo de práctica se llevó a cabo en su momento el centro de la agroindustria en Guatemala (Centro Agronómico Tropical de Investigación y Enseñanza CATIE, 2013).

De los modelos de gestión del conocimiento, el que se podría adaptar a las necesidades de los micronegocios y de la aplicación que usa la academia para poder gestionar el conocimiento hacia otras entidades de su entorno, es el modelo del proceso de creación del conocimiento de Nonaka y Takeuchi. Este tipo de modelo es dinámico sobre la creación del conocimiento que contextualiza tal proceso en un entorno social, dónde se produce la conversión de unos tipos de conocimiento en otros y que, por tanto, necesita de los individuos, pero sin circunscribirse a la individualidad, sino como un producto de la interacción entre personas (Aguilera-luque y Unibertsitatea, 2017).

Se considera como un activo intangible de una organización al aspecto del conocimiento, ya que se ha identifica como un elemento clave de las organizaciones y la sociedad para lograr ventajas competitivas, debido a su gran relevancia en el entorno organizacional, frente esta realidad, surge un nuevo enfoque dentro de la gestión empresarial conocido como la gestión del conocimiento. (Sánchez, 2005).

En el estudio realizado por Fuentes et al., (2016) menciona que las capacitaciones y nivel de escolaridad de los dueños de los negocios son los factores más destacados en la generación de ventajas competitivas, por lo que entre los factores intangibles como el conocimiento son de mucha importancia para generar ventaja competitiva en microempresas. Mediante el uso de este modelo se pretende derivar la utilización de este hacia cada dueño de un micronegocio, para que así posea los elementos que les faciliten la correcta gestión y manejo del negocio de manera eficiente.

\section{Gestión administrativa de los micronegocios}

La gestión administrativa y el manejo de micronegocios son procesos sencillos, a diferencia de las empresas grandes y medianas, las estructuras formales de organización altamente complejas no son necesarias en los pequeños negocios, como tal, para lograr determinar el rumbo del negocio, por lo general los micronegocios trabajan en condiciones informales los cuales no poseen una 
estructura definida, las estructuras organizacionales de los micronegocios se limitan a la necesidad y operaciones de los negocios.

La estructura que se utilizará en los procesos de manejo administrativo en las tiendas de barrio se basa en los formatos tradicionales de planes de negocios en los que constan 4 dimensiones; la educación financiera como esquema para el control interno, operacional, administrativo y mercadeo estratégico. Dimensiones que poseen la mayor parte de procesos que debe poseer un micronegocio. Se le denomina Riesgo al impacto y la probabilidad de que una amenaza o una serie de eventos puedan afectar de manera adversa la consecución de los objetivos, los riesgos a evaluar o determinar serán riesgos de tipo operativo debido a que la dimensión a evaluar es la gestión y manejo de micronegocios (Deloitte, 2015).

La fundamentación teórica del proyecto permite conocer la situación real del estudio, reflejando bajos índices en investigaciones de este tipo realizadas dentro del territorio ecuatoriano. El mayor número de investigaciones que se han realizado en este campo, proceden de países como Colombia y México, los cuales han permitido la elaboración de tesis, diagnósticos y guías para la implementación de estas, adaptadas en los pequeños negocios.

\section{Metodología}

La metodología empleada en el presente proyecto, cuenta con los siguientes aspectos, el enfoque es de la naturaleza mixta, como parte de la investigación- acción. Para el tipo de diseño se definió el carácter de la investigación como No experimental- transeccional, con un alcance de carácter descriptivo, lo cual permite describir en forma precisa sobre el desconocimiento por parte de los tenderos, con relación a los beneficios que ofrece la aplicación de herramientas administrativas de planificación estratégica.

\section{Figura 2}

\section{Metodología}

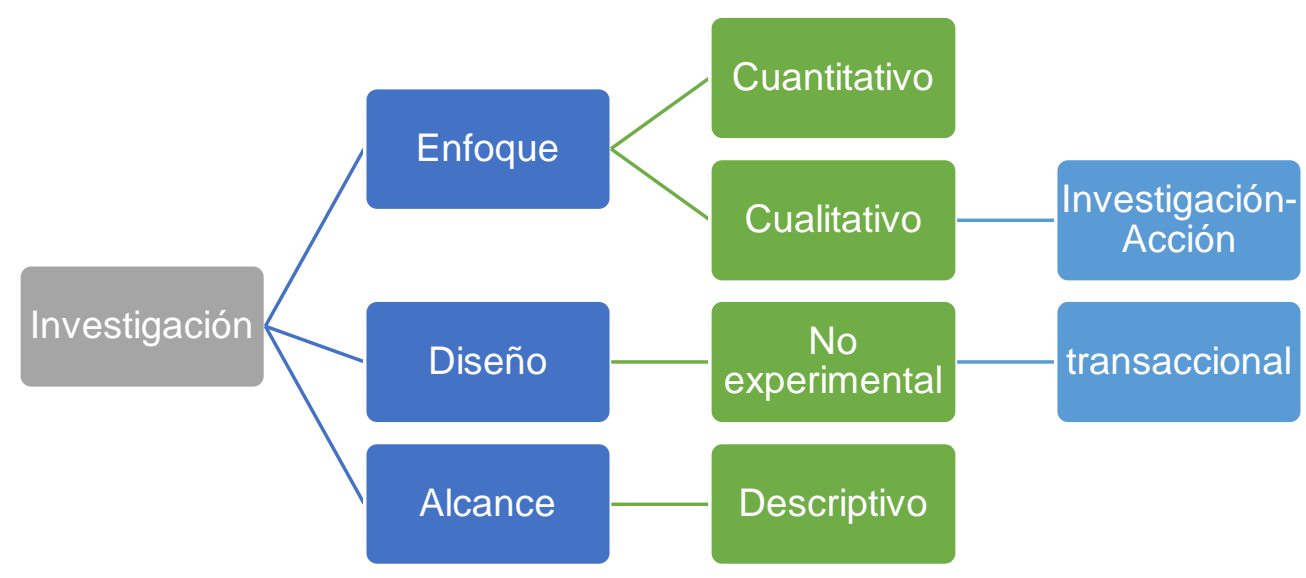




\section{Población y Muestra}

La población estudiada en la que se basó nuestro objeto de estudio sobre la gestión del conocimiento como herramienta para la gestión administrativa en el sector Guasmo Sur de Guayaquil, se consideró la población perteneciente al programa de tiendas que colaboran con la ONG Fundación Huancavilca, institución que facilitó la data que se registró en el año 2019, con 74 tiendas inscritas en el programa de vinculación con la comunidad en convenio con la Universidad de Guayaquil.

Arias et, al. (2016): "El muestreo intencional consiste en la selección por métodos no aleatorios de una muestra cuyas características sean similares a las de la población objetivo, en donde el investigador seleccione directa e intencionadamente los individuos de la población".

Para efectos del artículo, se consideró la muestra de 37 propietarios de tiendas de barrio para la realización de encuestas siendo este representativo al 50\% de la población total de 74 tiendas registradas en Fundación Huancavilca, la información que se menciona fue expedida por medios electrónicos, donde también consta el respectivo certificado con firma responsable de la dirección ejecutiva (Lcda. A. Molinero, comunicación personal, 5 de febrero del 2021) de la institución, documento que respalda la consolidación de los datos que faciliten el desarrollo y respaldo del proyecto de estudio.

\section{Figura 3}

Muestreo aplicado

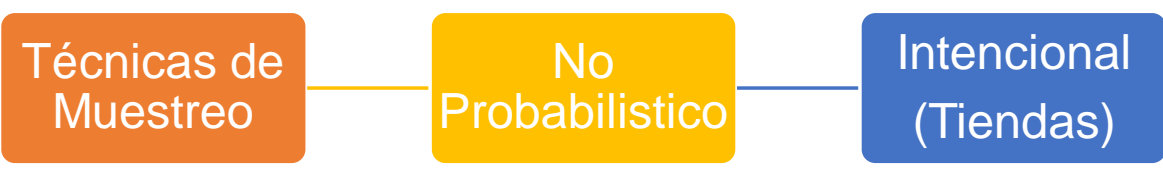

Tabla 2

Data de tiendas de barrio

\begin{tabular}{cc}
\hline & Tiendas de Barrio - Guasmo \\
\hline Población Total & 74 tiendas registradas \\
Muestra Intencional & 37 tiendas encuestadas \\
\hline
\end{tabular}

Nota. Elaboración propia datos obtenidos en Fundación Huancavilca por medio del programa de vinculación periodo, 2020.

Por otro lado, para lograr una riqueza en el análisis del problema planteado, se optó por añadir al estudio otros instrumentos de recolección de datos, con la finalidad de ampliar el conocimiento y análisis de los mismos, obteniendo así una visión del entorno de forma más integral y precisa. Las técnicas e instrumentos adicionales en la recolección de datos que utilizamos fueron entrevistas y observaciones dirigidos a los propietarios de tiendas sector Guasmo Sur a través de cuestionarios y ficha de observación. 


\section{Resultados y Discusión}

\section{Encuestas a Tiendas de Barrio}

Para el análisis de las encuestas dirigidas a los propietarios de tiendas de barrio sector Guasmo Sur, señalamos que para la recolección de los datos se utilizó muestro intencional, con datos parciales de 37 tiendas representativos al 50\% de la base de datos registradas en Fundación Huancavilca.

\section{Tabla 3}

\section{Escolaridad}

\begin{tabular}{lll}
\hline Variables & Frecuencia & \% \\
\hline Primaria & 10 & 27 \\
Secundaria & 11 & 30 \\
Superior & 16 & 43 \\
Ninguno & 0 & 0 \\
Total & $\mathbf{3 7}$ & $\mathbf{1 0 0}$
\end{tabular}

Nota. Elaboración propia datos obtenidos a través de la encuesta aplicada a los dueños de las tiendas de barrio Guasmo sur para definir el nivel de estudio, 2020.

En la tabla 5 se detalla el nivel de estudio que poseen los dueños de tiendas barriales sector Guasmo Sur, la cual muestra que un $43 \%$ corresponde a un grupo de tenderos que han cursado estudios superiores de forma incompleta, otro segmento de dueños de tiendas posee un $30 \%$ en nivel educativo secundario y un $27 \%$ cursó solo la primaria. Lo cual nos revela que al ser un total del $57 \%$ de personas que poseen estudios elementales entre la educación general básica y el bachillerato, son quienes se encuentran a cargo de la administración de estos negocios de manera empírica.

Dado el bajo nivel de escolaridad como indicador del poco conocimiento administrativo que poseen, siendo este el perfil académico que comparten los propietarios de tiendas. Es ineludible recalcar que la relación intrínseca que existe entre conocimiento y gestión administrativa, tal como lo apuntan varios escritores al ser este considerado como un requerimiento en la dirección y manejo de los negocios, así lo afirma Davenport (1998) en su libro Working knowledge: How organizations manage what they know, de Harvad Bussiness School Press, donde expone que los negocios que poseen un conocimiento práctico y científico en la materia como tal pueden entender como operar de manera eficiente.

\section{Tabla 4}

Edad

\begin{tabular}{ccc}
\hline Variables & Frecuencia & \% \\
\hline $30-40$ años & 23 & 62 \\
$41-50$ años & 9 & 24 \\
$51-60$ años & 5 & 14
\end{tabular}


Evelyn Verónica Madrid-Mosquera, Maribel Alexandra Clemente-González, Jhoanne Danniela Infante-Jaramillo y Estrella Zulema Rivera-Aguilar

ISSN 2477-9024. Innova Research Journal (Septiembre-Diciembre, 2021). Vol. 6, No. 3.1, pp. 19-40

\begin{tabular}{ccc}
\hline Variables & Frecuencia & $\%$ \\
\hline 61 o más & 0 & 0 \\
Total & 37 & 100 \\
\hline
\end{tabular}

Nota. Elaboración propia datos obtenidos a través de la encuesta aplicada a los dueños de las tiendas de barrio Guasmo sur para definir el rango de edad, 2020.

La edad promedio entre los dueños que dirigen las tiendas de barrio sector Guasmo Sur, según los registros de la tabla 6 proyectan que un $62 \%$ son personas que están en un rango de 30 a 40 años, un $24 \%$ se encuentran entre los 41 a 50 años y un $14 \%$ pertenece al grupo de personas con edades que oscilan entre los 51 a 60 años.

Entre los parámetros de medición generales de las personas que se encuentran a cargo de los micronegocios podemos verificar que la edad promedio entre los tenderos, se encuentran comprendidos entre los 30 a 40 años, datos que en paralelo a otros análisis que se han realizado en este tipo de investigaciones publicado por la revista de economía del Rosario, para Alvarado et al., (2018) la información comparte el criterio de medición debido a que los resultados reflejaron "que la mayoría de los microempresarios encuestados reportó tener más de 41 años de edad con un 57.6\% de la población de micronegocios en México".

Esta característica permite afirmar que los negocios son coordinados por personas que se encuentran en una etapa de madurez y con varias responsabilidades a su cargo ya sean estos a nivel personal u otros aspectos sean estos sociales, económicos y afectivos, comprendidos entre 40 años en adelante.

\section{Tabla 5}

\section{Género}

$\begin{array}{ccc}\text { Variables } & \text { Frecuencia } & \% \\ \text { Femenino } & 23 & 64 \\ \text { Masculino } & 14 & 36 \\ \text { Total } & 37 & 100\end{array}$

Nota. Elaboración propia datos obtenidos a través de la encuesta aplicada a los dueños de las tiendas de barrio Guasmo sur para establecer el género, 2020.

Según las respuestas arrojadas en la tabla 7 el género que predomina en la dirección de los negocios "tiendas de barrio" sector Guasmo Sur en Guayaquil; el 64\% pertenece al género femenino y el 36\% restante al género masculino. Estos datos pueden ser contrastados con el estudio de Alvarado et al., (2018) quien señala lo siguiente: "Es conveniente destacar que los datos fueron recabados casi de manera equitativa entre hombres $(48,5 \%)$ y mujeres $(51,5 \%)$ en la administración de un micronegocio familiar en México".

Dejando establecido las semejanzas de resultados existentes en la comparativa de ambos procesos planteados, sobre cuál es el género que se más se rige dentro de la administración de estos negocios, tanto en el Guasmo Sur de Guayaquil como los referidos de la ciudad de México. Resaltamos de manera objetiva el hallazgo de similitudes en ambas evaluaciones efectuadas, ante la notoriedad del alto porcentaje en las frecuencias del género femenino en términos generales. 
Por otro lado, la diferencia en cuanto a la presencia masculina frente a la femenina en la gerencia de las despensas, no pasa desapercibido en ninguno de los procesos estadísticos mostrados, a causa de que los porcentajes de participación entre ambos géneros en la gestión de las unidades productivas informales son significativos.

\section{Tabla 6}

Factor de impedimento para capacitación de tenderos

\begin{tabular}{lcc}
\hline \multicolumn{1}{c}{ Variables } & Frecuencia & \% \\
\hline Tiempo & 22 & 60 \\
Dinero & 6 & 16 \\
No tiene ayudante & 3 & 8 \\
No está interesado & 2 & 5 \\
Innecesario & 4 & 11 \\
Total & $\mathbf{3 7}$ & $\mathbf{1 0 0}$ \\
\hline
\end{tabular}

Nota. Elaboración propia datos obtenidos a través de la encuesta aplicada a los dueños de las tiendas de barrio Guasmo sur acerca de las razones por las que no asisten a capacitaciones sobre ventas u otras áreas, 2020.

Entre los factores que causan diversos impedimentos para la capacitación dirigido a los tenderos según las respuestas obtenidas en la tabla 8 son; factor tiempo con un $60 \%$, el aspecto económico con el $16 \%$, el criterio de la poca necesidad de los tenderos en obtener una capacitación con $11 \%$, la falta de un asistente o ayudante con $8 \%$ y en última instancia el $5 \%$ restante no posee ningún tipo de interés al respecto.

\section{Tabla 7}

Documentación legal para la operación de tiendas

\begin{tabular}{lccc}
\hline Variables & Frecuencia & Cálculo & $\%$ \\
\hline RISE & 10 & $10 / 37$ & 27 \\
RUC & 15 & $15 / 37$ & 40.5 \\
Uso de suelo & 6 & $6 / 37$ & 16 \\
Bomberos & 6 & $6 / 37$ & 16 \\
Permiso Municipal & 8 & $8 / 37$ & 22 \\
IESS & 2 & $2 / 37$ & 5 \\
Ninguno & 12 & $12 / 37$ & 32 \\
\hline
\end{tabular}

Nota. Elaboración propia datos obtenidos a través de la encuesta aplicada a los dueños de las tiendas de barrio Guasmo sur acerca del marco legal bajo el que están regidos, 2020.

En la tabla 9 el estudio con respecto a la documentación legal de las 37 tiendas de barrio del sector del Guasmo Sur; 15 de ellas poseen RUC representando el 40,5\%, Otras 10 poseen RISE lo cual es representativo al $27 \%$ y 12 no poseen algún tipo de documentación con un $32 \%$.

De las tiendas que respondieron que poseen documentación; 6 establecimientos indicaron que adicional tienen el respectivo permiso de uso de suelo siendo el 16\%; Otros 6 negocios poseen 
permiso de bomberos correspondiente a un $16 \%$. Por otra parte 8 tiendas barriales mencionaron que, si tienen permiso de funcionamiento municipal con un $22 \%$. Finalmente, solo 2 tiendas tenían constancias de su respectiva documentación con el IESS siendo 5\% de minoría en comparación a otras tiendas, debido a la particular que estos dos últimos negocios compartían tenían a su disposición un colaborador a su cargo.

Por los datos expuestos notamos que revelan el factor común en cuanto a la escaza estructura formal en lo que concierne al aspecto legal, dando lugar a lo que se conoce popularmente como negocios informales. Tradicionalmente el denominado sector informal, debido a la escasa productividad, incapacidad de brindar protección social a los trabajadores. El desafío de estos negocios más o menos abierto al Estado y la cercanía que estos mantienen con actividades propiamente ilícitas, por su carácter de informalidad sean aspectos legales y/o administrativos, lo cual no es visto con buenos ojos, pero, ha sido tolerado por considerarse como un mal menor, al ser esto preferible frente a la realidad de desempleo abierto en el que se encuentran inmersos los individuos en una sociedad en la que coexisten mutuamente. (Tokman, 2004).

Cabe agregar que un proceso de formalización completo de un micronegocio implica más que sólo el registro ante las entidades regulatorias. Una microempresa para ser totalmente formal debe cumplir con obligaciones que van más allá del pago de impuesto, debido a que los procesos de registro de las microempresas ante el régimen fiscal es un tanto ambiguo: por un lado, se facilita el registro a internet, por otro se complejiza el proceso de tributación dentro del mismo. (Rivera et al., 2016)

\section{Tabla 8}

Motivos para tener una tienda

\begin{tabular}{ccc}
\hline Variables & Frecuencia & \% \\
\hline Falta empleo & 8 & 22 \\
Mejorar ingresos & 14 & 38 \\
Negocio familiar & 5 & 13 \\
No depender de un jefe & 10 & 27 \\
TOTAL & $\mathbf{3 7}$ & $\mathbf{1 0 0}$
\end{tabular}

Nota. Elaboración propia datos obtenidos a través de la encuesta aplicada a los dueños de las tiendas de barrio Guasmo sur para conocer los motivos de creación de las tiendas, 2020.

El motivo por el cual los dueños de tiendas iniciaron este tipo de negocio como se indica en la tabla 10 son: un $38 \%$ de ellos invirtieron en este tipo de emprendimiento para lograr tener mejoras en el ingreso de su hogar, continuo a eso el $27 \%$ manifestaron que para no querían tener que depender de un jefe, el $22 \%$ por la falta de empleo y un $13 \%$ debido a la tradición de continuar con los negocios familiares.

En el estudio efectuado por Alvarado et al., (2018) muestran los porcentajes de micronegocios según los motivos por los que fueron emprendidos, publicados en la revista económica del Rosario que, de manera general, se aprecia que, el motivo más frecuente para 
emprender un micronegocio fue "mejorar el ingreso familiar" y "complementar el ingreso familiar", mientras que el de menor importancia fue "no tener la escolaridad requerida para un empleo".

\section{Tabla 9}

Ingresos extras

\begin{tabular}{ccc}
\hline Variables & Frecuencia & $\%$ \\
\hline Sí & 17 & 46 \\
No & 20 & 54 \\
Total & $\mathbf{3 7}$ & $\mathbf{1 0 0}$ \\
\hline
\end{tabular}

Nota. Elaboración propia datos obtenidos a través de la encuesta aplicada a los dueños de las tiendas de barrio Guasmo sur acerca de otros medios de obtener ingresos, 2020.

Si consideramos los ingresos adicionales como alternativas para poder complementar las actividades económicas que realizan en las tiendas de barrio, notamos que en la tabla 11 el $54 \%$ de propietarios respondieron que no realizaban algún tipo de actividad para generar ingresos extras dentro de sus negocios y un $46 \%$ indicaron que sí realizaban una actividad como tal, que diera soporte a sus actividades comerciales dentro de las tiendas de barrio.

\section{Tabla 10}

Tiempo de apertura del negocio

\begin{tabular}{ccc}
\hline Variables & Frecuencia & \% \\
\hline Menor a 1 año & 10 & 27 \\
1-3 años & 17 & 46 \\
4-6 años & 4 & 11 \\
7 o mas & 6 & 16 \\
Total & $\mathbf{3 7}$ & $\mathbf{1 0 0}$ \\
\hline
\end{tabular}

Nota. Elaboración propia datos obtenidos a través de la encuesta aplicada a los dueños de las tiendas de barrio Guasmo sur para conocer el tiempo de funcionamiento de las tiendas, 2020

El Telégrafo (2020) expresó que los emprendimientos son negocios que funcionan hasta tres años, nueve de cada 10 mueren. La mayoría de las tiendas de barrio tienen una administración informal o empírica, este factor puede ser uno de los causales de no permanecer, debido al desconocimiento de procedimientos administrativos. Considerando el argumento postulado, referimos las estadísticas analizadas sobre el lapso de tiempo que llevan desempeñándose las unidades productivas informales "tiendas de barrio" sector Guasmo, los resultados obtenidos en la tabla 12 apuntan que la mayoría de despensas con un $46 \%$ han tenido un promedio de vida de 1 a 3 años, otro grupo de negocios manifestaron que su tiempo de vida es "menor a 1 año" con el 27\%, el 16\% de negocios ha durado en promedio de "7 a más años", y el 11\% sobrante revelo que el lapso de vida ha sido alrededor de 4 a 6 años.

Para Barzola (2015): "Este tipo de negocios que son creados empíricamente con la necesidad de generar ganancias diarias para el inversor, no suelen tener un seguimiento o estudio previo para 
Evelyn Verónica Madrid-Mosquera, Maribel Alexandra Clemente-González, Jhoanne Danniela Infante-Jaramillo y Estrella Zulema Rivera-Aguilar

ISSN 2477-9024. Innova Research Journal (Septiembre-Diciembre, 2021). Vol. 6, No. 3.1, pp. 19-40

reconocer sus fortalezas, amenazas, debilidades y oportunidades", lo cual sería la causa del problema del cierre o fracaso total de la inversión.

\section{Tabla 11}

Responsable de la atención de tiendas

\begin{tabular}{ccc}
\hline Variables & Frecuencia & \% \\
\hline Dueño de tienda & 28 & 76 \\
Familiares & 8 & 21 \\
Empleado & 1 & 3 \\
TOTAL & $\mathbf{3 7}$ & $\mathbf{1 0 0}$ \\
\hline
\end{tabular}

Nota. Elaboración propia datos obtenidos a través de la encuesta aplicada a los dueños de las tiendas de barrio Guasmo sur para definir quiénes están a cargo, 2020.

Las personas que son responsables por la atención de las tiendas barrio sector Guasmo Sur, según los datos arrojados en la tabla 13 un $76 \%$ son los propietarios, otro segmento indica que el $21 \%$ son familiares quienes colaboran con la atención al público y un $3 \%$ tienen personal encargado

\section{Tabla 12}

Registros Contables

\begin{tabular}{ccc}
\hline Variables & Frecuencia & \% \\
\hline Cuaderno & 14 & 38 \\
Computadora & 9 & 24 \\
Mentalmente & 6 & 16 \\
Ningún registro & 8 & 22 \\
Total & $\mathbf{3 7}$ & $\mathbf{1 0 0}$ \\
\hline
\end{tabular}

Nota. Elaboración propia datos obtenidos a través de la encuesta aplicada a los dueños de las tiendas de barrio Guasmo sur sobre el manejo contable que efectúan, 2020.

La tabla 14 puntualiza cuál es el proceso de registros contables, utilizados por estos negocios un $38 \%$ lo hace de manera manual en un cuaderno, el $24 \%$ lleva registros en equipos de cómputo, un $22 \%$ no realiza ningún registro y el $16 \%$ realiza el proceso de forma mental.

\section{Tabla 13}

Medios de financiamiento

\begin{tabular}{ccc}
\hline Variables & Frecuencia & \% \\
\hline Préstamo bancario & 7 & 19 \\
Préstamo cooperativo & 2 & 6 \\
Préstamo familiar & 10 & 27
\end{tabular}




\begin{tabular}{ccc}
\hline Variables & Frecuencia & $\%$ \\
\hline Préstamo informal & 2 & 5 \\
Ahorros & 16 & 43 \\
Total & 37 & 100 \\
\hline
\end{tabular}

Nota. Elaboración propia datos obtenidos a través de la encuesta aplicada a los dueños de las tiendas de barrio Guasmo sur sobre sus medios para emprender, 2020.

En la tabla 15 observamos que el capital con el que inician los negocios de tiendas de barrio obtuvieron financiación mediante ahorros según el $43 \%$ de respuestas, otro segmento respondió que la financiación obtenida fue por préstamo familiar con $27 \%$, otro grupo con el 19\% adquirió un préstamo bancario para iniciar sus operaciones comerciales, un 6\% mencionó que recurrieron a préstamos de cooperativa y el $5 \%$ restante acudieron a prestamistas informales.

Para Alvarado et al., (2018) Estos autores señalan que las tres fuentes de financiamiento iniciales de un empresario son los recursos propios o de la familia, de socios y de amigos. Por lo tanto, las perspectivas de crecimiento de los micronegocios familiares son limitadas, dado que no se invierte en capital físico (infraestructura, maquinaria, herramienta, etcétera), debido a que a la mayoría de los microempresarios no le interesa obtener apoyo económico de alguna entidad financiera. Determinando que este giro de micronegocios funciona en su mayoría de manera autónoma con respecto a las financiaciones de sus propios recursos, pese a las pocas oportunidades que se les presentan en el sector financiero.

\section{Entrevistas a Tiendas de Barrio}

Para efectos de la investigación sobre la gestión del conocimiento como herramienta para la gestión administrativa en el sector Guasmo Sur, se consignaron técnicas de entrevistas y observaciones al $10 \%$ de la población total de los micronegocios "tiendas de barrio" del sector, en base a los datos emitidos por Fundación Huancavilca, para el desarrollo del proyecto.

\section{Tabla 14}

Estrategias de Publicidad, Descuentos y Promociones

\begin{tabular}{ccc}
\hline Variables & Frecuencia & $\%$ \\
\hline Sí & 2 & $29 \%$ \\
No & 5 & $71 \%$ \\
Total & 7 & $100 \%$ \\
\hline
\end{tabular}

Nota. Elaboración propia datos obtenidos a través de entrevista aplicadas a propietarios de tiendas de barrio del Guasmo Sur.

Los tenderos respondieron en la tabla 16 de entrevistas que no realizan estrategias de publicidad, descuento o promoción para atraer clientela en un $71 \%$ porque no tiene variedad de productos o desconoce cómo poder hacerlo, frente a un $29 \%$ quienes si utilizan algún tipo de estrategia de venta. 
Evelyn Verónica Madrid-Mosquera, Maribel Alexandra Clemente-González, Jhoanne Danniela Infante-Jaramillo y Estrella Zulema Rivera-Aguilar

ISSN 2477-9024. Innova Research Journal (Septiembre-Diciembre, 2021). Vol. 6, No. 3.1, pp. 19-40

\section{Tabla 15}

Existencia de herramientas de control

\begin{tabular}{llll}
\hline \multicolumn{1}{c}{ Variables } & & Frecuencia & $\%$ \\
\hline Sí & 2 & $29 \%$ \\
No & 5 & $71 \%$ \\
Total & 7 & $100 \%$ \\
\hline
\end{tabular}

Nota. Elaboración propia datos obtenidos a través de entrevista aplicadas a propietarios de tiendas de barrio del Guasmo Sur, 2020 .

En las entrevistas realizadas que se detalla en la tabla 17 los administradores del sector informal tiendas de barrio indican que un $29 \%$ si cuentan con alguna herramienta que les permita organizar y llevar el avance de su negocio y $71 \%$ restante de tiendas, expreso que no, ya que sólo con las facturas emitidas por los proveedores tratan de controlar el stock de mercadería, por lo pequeño que es el negocio y creen que no tienen que llevar registros contables.

La provincia del Guayas según Chávez et al., (2018) tiene una alta concentración de pequeñas y microempresas, conociendo que estas generan un alto uso de mano de obra, son vitales en la reducción del desempleo, en el dinamismo económico provincial y en la estabilidad social y política que de ello se deriva, que este tipo de empresas, son pilares en la innovación. Considerando que son estructuras organizativas flexibles con alta capacidad de innovación por la estrecha relación con los clientes, que les permite adaptarse a entornos cambiantes como los actuales tiempos, en donde los dominios de las tecnologías predominan y son herramientas que permiten tener un mejor control del emprendimiento y facilitar la interacción con los clientes, consecuente a esto transforma la accesibilidad de los bienes y servicios que ofrecen.

\section{Tabla 16}

Oportunidad de capacitación

\begin{tabular}{lll}
\hline Variables & Frecuencia & \% \\
\hline Si & 0 & $0 \%$ \\
No & 7 & $100 \%$ \\
Total & $\mathbf{7}$ & $\mathbf{1 0 0}$
\end{tabular}

Nota. Elaboración propia datos obtenidos a través de las entrevistas aplicadas a los dueños de tiendas de barrio, sobre la oportunidad de capacitación, 2020.

La orientación emprendedora individual (OEI) son aquellas características o actitudes personales subyacentes que poseen los individuos que los llevan a emprender actividades emprendedoras (Bolton y Lane, 2012)

Por medio de las entrevistas realizadas, en la tabla 18, se visualiza que en la totalidad el $100 \%$ de los casos, los tenderos no han tenido a su disposición la oportunidad de recibir capacitación para mejorar la administración de sus negocios, sean estos con proveedores, fundaciones, centros 
de capacitación, u otros medios. En contexto al no existir una atención directa que busque la capacitación a los administradores de tiendas de barrio, sector informal del Guasmo Sur, no se podrán desarrollar habilidades técnicas requeridas para el crecimiento de los mismos., en muchas ocasiones mencionan que tratan de autocapacitarse, pero que al no tener facilidades de tener a un referido o una persona experta para realizar consultas y despejar dudas sobre diversas áreas de desconocimiento que poseen, se ven impedidos en tomar acciones de innovación por miedos a fracasar en sus emprendimientos.

Es importante resaltar la necesidad de orientación que solicitan los tenderos, dado que existe una demanda que no está siendo atendida a este sector informal, en base a los resultados obtenidos en las estadísticas realizadas (revisar taba 8) y que muestran que solo una minoría tendrían renuencia por capacitarse frente a la mayoría que posee ciertos impedimentos pero que en las entrevistas han dado a conocer su deseo por adquirir conocimiento sobre procesos administrativos entre otros.

\section{Observaciones a Tiendas de Barrio}

Posterior a las entrevistas, como parte del proceso final de la recolección de datos para el análisis situacional de las tiendas, encontramos la siguiente información relevante, en las observaciones realizadas a los micronegocios.

\section{Tabla 17}

Aspectos físicos

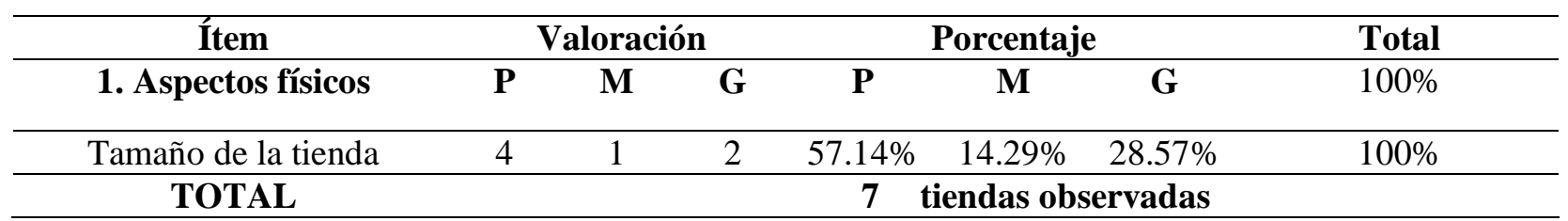

Nota. Elaboración propia, datos obtenidos a través de los resultados de la ficha de observaciones realizadas a los dueños de tiendas del Guasmo Sur, 2020.

Examinando los resultados obtenidos en la tabla 19, por medio de las observaciones realizadas en cuanto aspecto físico que poseen las despensas del sector del Guasmo Sur el 57.14\% se definen como pequeñas, el siguiente segmento con un $14.29 \%$ corresponden a una valoración de tiendas medianas y un $28.57 \%$ restante de los negocios analizados entran en la categoría de gran dimensión dado el tamaño de la tienda adicional a este aspecto le siguen otros factores como el capital, el volumen de ventas, número de trabajadores, entre otros. Es así que para Haro (2021) en su artículo recalca en que la empresa pequeña es más productiva en comparación a otros negocios de mayor dimensión, ya que a un mayor tamaño más altos son los costos y la posibilidad de cometer errores, por lo que es necesario observar y plantear incidencias que permitan categorizar diferentes cuestiones con respecto a la liquidez, la eficiencia técnica, la eficacia en la consecución de beneficios y la gestión ejecutada en los micronegocios. 
Evelyn Verónica Madrid-Mosquera, Maribel Alexandra Clemente-González, Jhoanne Danniela Infante-Jaramillo y Estrella Zulema Rivera-Aguilar

ISSN 2477-9024. Innova Research Journal (Septiembre-Diciembre, 2021). Vol. 6, No. 3.1, pp. 19-40

\section{Tabla 18}

Equipamiento del negocio

\begin{tabular}{lccccccc}
\hline \multicolumn{1}{c}{ Ítem } & \multicolumn{2}{c}{ Valoración } & \multicolumn{2}{c}{ Porcentaje } & Total \\
\hline Equipamiento & NT & TP & TS & NT & TP & TS & \\
Caja registradora & 6 & 0 & 1 & $85.71 \%$ & $0.00 \%$ & $14.29 \%$ & $100 \%$ \\
Letrero, identificación del negocio & 5 & 0 & 2 & $71.43 \%$ & $0.00 \%$ & $28.57 \%$ & $100 \%$ \\
Luminarias & 0 & 4 & 3 & $0.00 \%$ & $57.14 \%$ & $42.86 \%$ & $100 \%$ \\
Productos en estanterías, perchas & 0 & 5 & 2 & $0.00 \%$ & $71.43 \%$ & $28.57 \%$ & $100 \%$ \\
Frigoríficos en funcionamiento & 1 & 4 & 2 & $14.29 \%$ & $57.14 \%$ & $28.57 \%$ & $100 \%$ \\
Total & & & & $\mathbf{7}$ tiendas observadas & \\
\hline
\end{tabular}

Nota. Elaboración propia, datos obtenidos a través de los resultados de la ficha de observación, ítem equipamiento de las tiendas de barrio del Guasmo sur, 2020.

Naranjo (2018): "La adecuación de las tiendas populares de los beneficiarios directos del proyecto no se efectúa en formatos comerciales estandarizados". El autor indica que este tipo de tiendas no manejan un bosquejo organizado en base a sistemas de gestión, marketing, y otros de forma orgánica que satisfaga completamente las necesidades de potenciales clientes esquematizados comercialmente.

Referente al equipamiento del negocio en la tabla 20 tenemos en consideración varios aspectos a evaluarse dentro de las despensas observadas, en un primer aspecto considerando el $85.71 \%$ de los establecimientos no existe el manejo de una caja registradora, lo cual se traduce en una ralentización de los procesos contables al momento de llevar un control o registro de ventas, transacciones realizadas, generando a su vez un desconocimiento en las entradas y salidas de mercancías además de los flujos de ingresos y egresos del circulante que genera el negocio en sí. Solo el $14.29 \%$ de estas tiendas tienen el equipo suficiente. Si bien examinamos otras variables de los micronegocios el $71.43 \%$ de los establecimientos no tienen identificación respectiva que permitan diferenciarse de la competencia dada la informalidad en la que operan. Rudimentario

Un elemento importante como lo es la iluminación de las despensas presenta un $57.14 \%$ sin mucha acogida en la implementación de los micronegocios frente al $42.86 \%$ de tiendas que si poseen la iluminaria suficiente en su local, esto a su vez, va de la mano con la visualización de los productos que se comercializan ya que en otra instancia se refleja que el $71.43 \%$ de los tenderos tienen pocos productos en estanterías y perchas, no obstante, un $28.57 \%$ tiene los productos suficientes organizados en las perchas y estanterías de su dependencia. Como último ítem respecto al uso de frigoríficos una minoría con el $14.29 \%$ no posee este tipo de herramienta de trabajo, un $57.14 \%$ exterioriza pocos equipos de refrigeración para sus productos y un $28.57 \%$ tiene el equipo suficiente en funcionamiento en el área de comercialización. 


\section{Conclusiones}

Para la conducción de conclusiones sesudas, señalamos que, las evaluaciones a los datos que fueron presentados con anterioridad, se relacionan con los fundamentos planteados en la investigación elaborada por Edith Navarrete y Edgar Sansores (2011) de la Universidad de Quintana Roo, donde mencionan cuáles son las causales de quiebra en los micronegocios (tiendas esquineras), debido a la serie de obstáculos que dificultan su supervivencia y crecimiento. Caracterizadas por una escasa formación en el desarrollo de las habilidades empresariales, con poca información del conocimiento sobre el mercado, problemas de comercialización, falta de vinculación con programas para el desarrollo e innovación tecnológica, difícil acceso a esquemas de financiamiento ofrecidos por la banca comercial, y, en general, esa situación es la encargada de provocar un alto índice de mortandad en las pequeñas empresas durante los dos primeros años de operación, traducido en una inestabilidad económica y pérdida de empleos en la entidad.

Basándonos en el objetivo principal sobre de la situación actual que presentan las tiendas de barrio sector Guasmo Sur, según los datos que corresponden a encuestas, entrevistas y observaciones aplicados a los dueños de tiendas, podemos señalar que el $97 \%$ son dirigidos por el núcleo familiar; compuesto en un $76 \%$ de respuestas por los tenderos, un $21 \%$ conformado por la asistencia de algún familiar cercano en el manejo del negocio, autodenominándose así como negocios familiares, por la mayoría de resultados. El porcentaje restante indicó tener a su cargo un colaborador externo bajo contrato, siendo así una minoría respectiva a un 3\% de tiendas estudiadas.

Otro dato relevante es que el 45,9\% de estos negocios son única fuente de ingresos para sus familias debido a que no realizan otro tipo de actividades que les genere ingresos extras. El nivel de estudio de los tenderos es en promedio de nivel medio, tomando en consideración que dentro de la consulta se hallaron personas con estudios superiores incompletos lo cual corresponde al 43,2\% grupo, se les podría facilitar la comprensión del uso de tecnología, debido a que sólo el $24 \%$ de tenderos usan equipos tecnológicos destinado a las operaciones del negocio. Comprobando así que los micronegocios, no poseen los equipos suficientes para la atención al cliente, frente a la gran demanda que los consumidores requieren en los tiempos actuales.

Independientemente del tamaño del negocio, ya sea por la dimensión del capital, recurso humano o material, no existen tamaños pequeños e insignificantes que no necesitasen llevar una dirección, un orden, un control, un plan que aporte al crecimiento de este. Dejando así constancia sobre la situación actual que presentan las tiendas de barrio del sector Guasmo Sur, por medio de los datos compilados, concluyendo así la existencia de diversos requerimientos necesarios, de forma inmediata, en la implementación de estrategias y recursos dentro de los micronegocios mencionados, para así poder alcanzar una mejora con respecto a la situación actual en la que se encuentran inmersos las tiendas de barrio del sector Guasmo Sur. Factores como el desconocimiento de procesos administrativos, contables, entre otros, afectan de manera negativa a las tiendas de barrio poniéndolas en riesgo.

\section{Recomendaciones}

Se recomienda que, a través de programas de asesoría impulsadas por instituciones académicas en general, puedan destinar parte de sus actividades en sus labores vinculación con la 
comunidad del Guasmo Sur, para lograr la capacitación y gestión del conocimiento en las micronegocios o microempresas, especialmente a los propietarios de tiendas de barrios, para el progreso de estos y que además pueda darse seguimiento de estos programas.

Realizar seguimiento, al ciclo de vida de las tiendas de forma periódica, para observar la tendencia de los procesos aplicados, conocer el comportamiento y los resultados que han generado. El uso de la tecnología en los actuales tiempos es un recurso oportuno en la comunicación y administración de un negocio sea comercial o de servicio en donde, el propietario de una tienda de barrio puede sacar provecho a esta herramienta para poder capacitarse y además poder facilitar y agilizar la organización de su negocio.

Gestionar convenios con otras instituciones o fundaciones, que permitan expandir este tipo de estudio en otras zonas de vulnerabilidad, que requieran de formación y aprendizaje en áreas administrativa y financiera, para el impulso que requieren muchos negocios informales o en vías de desarrollo, para su fortalecimiento.

\section{Limitaciones}

Entre las limitaciones encontradas en el desarrollo del estudio, se menciona la presencia de la pandemia mundial por Covid-19 que se atravesó en sus primeras fases y que aún se mantiene dentro del entorno en los actuales tiempos, lo cual dificulto parcialmente la obtención de datos de forma física, dada la implementación de los adecuados procesos de restricciones en la movilización, cuarentenas obligatorias y aplicación de los protocolos de bioseguridad, distanciamiento social y cumplimiento de los aforo emitidos por la COE nacional en conjunto con la municipalidad.

\section{Referencias Bibliográficas}

Aguilera-luque, A. M., \& Unibertsitatea, M. (2017). La gestión del conocimiento organizacional. Research Gate, (January). https://doi.org/10.13140/RG.2.2.28487.29600

Alvarado, L. E., Rodríguez, J. O., y Ramírez, D. M. (2018). Factores que influyen en el emprendimiento de un micronegocio familiar en México. Revista de Economía del Rosario, 21(1), 153-180. https://doi.org/10.12804/revistas.urosario.edu.co/economia/a.6816

Alvarado Lagunas, E., Dávila Aguirre, M. C., \& Vázquez Zacarías, M. A. (2018). Factors that influence the continuity and survival of a micro-business in Mexico. Contaduría y administración, 63(3), 24-25. http://doi.org/10.22201/fca.24488410e.2018.1281

Arias, G. J., Villasís, K. M., y Miranda, N. M. (2016). El protocolo de investigación III: la población de estudio. Revista Alergia México, 63(2), 201-206. https://www.redalyc.org/articulo.oa?id=486755023011

Centro Agronómico Tropical de Investigación y Enseñanza CATIE. (2013). Gestión del conocimiento para la innovación del desarrollo rural sostenible en Guatemala: fortaleciendo la agricultura familiar y la economía. Guatemala.

Chávez Cruz, G., Campuzano Vásquez, J., y Betancourt Gonzaga, V. (2018). Las Micro, Pequeñas y Medianas Empresas. Clasificación para su estudio en la carrera de Ingeniería en 
Contabilidad y Auditoría de la Universidad Técnica de Machala. Revista Conrado, 14(65), 247-255. http://conrado.ucf.edu.cu/index.php/conrado

Código Orgánico de la Producción, Comercio e Inversiones (2010, 29 de diciembre). Art. 53. Del Fomento y Desarrollo de las Micro, Pequeñas y Medianas Empresas (MIPYMES). https://www.correosdelecuador.gob.ec/wpcontent/uploads/downloads/2018/11/COPCI.pdf

Davenport, T. H., \& Prusak, L. (1998). Working knowledge: How organizations manage what they know. Harvard Business Press. https://bit.ly/3pj3gde

Deloitte. (2015). Evaluación de Riesgos. https://bit.ly/3w1sA8F

Fuentes, N., Osorio, G., y Mungaray, A. (2016). Capacidades Intangibles para la competitividad Microempresarial en México. Problemas del Desarrollo, 47(186), 83-106. https://doi.org/10.1016/j.rpd.2016.03.003

Forcadell, F.J., y Úbeda, F. (2021). La orientación emprendedora del fundador y el éxito en la internacionalización de la empresa. Emprendimiento y Negocios Internacionales. https://doi.org/10.20420/eni.2021.425

Galeano, M. E. (2020). Diseño de proyectos en la investigación cualitativa. Colombia: Universidad Eafit.

Giraldo, O. C. (2018). La gestión del conocimiento en las organizaciones y las regiones: una revisión de la literatura. Tendencias, 19(1), 4016. http://doi.org/10.22267/rtend.181901.91

González, P. C., Agudelo, C., y Ortiz, O. (2020). Estrategias comerciales implementadas por las tiendas de barrio frente a la llegada de las tiendas de descuento del sector laureles de Medellín. Tecnológico de Antioquia Institución Universitaria. https://dspace.tdea.edu.co/handle/tdea/757

Haro Sarango, A. F. (2021). El tamaño de la empresa y su influencia en la productividad del sector comercio. INNOVA Research Journal, 6(3), 227-245. https://doi.org/10.33890/innova.v6.n3.2021.1781

Bartesaghi, A., de Souza Silveira Greco, S. M., Lasio, M.V., Varela, R., Veiga, L., Kew, P. y Herrington, M. (2016). GEM América Latina y el Caribe 2015. Global Entrepreneurship Research Association. https://www.gemconsortium.org/file/open?fileId=49822

Hidalgo, A. (29 de enero de 2017). El telégrafo. El Guasmo hacienda que se convirtió en una microciudad. https://bit.ly/3BSFHLH

INEC. (2010). Instituto Nacional de Estadística y Censos. Obtenido de https://bit.ly/3BXMbZV

La Vitrina (2019). Experiencias de campo del proyecto de investigación para tenderos. Cuadernos del semillero de investigación "La Vitrina". Documentos De Trabajo Areandina, (1). https://doi.org/10.33132/26654644.1463

Líderes. (4 de julio de 2016). Las tiendas de barrio desarrollan estrategias. Recuperado el 22 de febrero de 2021, de revistalideres.ec: https://www.revistalideres.ec/lideres/tiendasdebarrioestrategias-innovacion-negocios.html

Londoño Toro, C. M. (2018). Caracterización de los negocios barriales en los barrios del borde oriental de la comuna 10 de Medellín. Revista CIFE: Lecturas De Economía Social, 20(33), 95-116. https://doi.org/10.15332/22484914.4879

Ministerio de Industria y Productividad (2019). Reglamento de inversiones del código orgánico de la producción. https://bit.ly/3nGZV55

Mora-Riapira, E. H., Vera-Colina, M. A., \& Melgarejo-Molina, Z. A. (2015). Planificación estratégica y niveles de competitividad de las Mipymes del sector comercio en Bogotá. Estudios gerenciales, 31(134), 79-87. https://doi.org/10.1016/j.estger.2014.08.001 
Navarrete Marneou, Edith and Sansores Guerrero, Edgar, Quintana Roo Mexico Micro, Small and Medium Sized Business Failure: A Multi-Variable Analysis (2011). Revista International Administración \& Finanzas, 4(3), 21-33, 2011, https://ssrn.com/abstract=1884519

Naranjo-Silva, E. R., Zabala-Jarrín, H. A., Gómez-Acosta, M. I., y Acevedo-Suárez, J. A. (2018). Estrategias de Packing en la red de tiendas populares en Riobamba. Ingeniería Industrial, 39(2), 204-214. http://scielo.sld.cu/pdf/rii/v39n2/1815-5936-rii-39-02-204.pdf

Páramo, D., García, O. L., y Arias, M. O. (2011). Hacia una tipología de tenderos de Manizales. Pensamiento \& Gestión [Escuela de negocios Universidad del Norte], 28, 196-216.

Páramo, D. (2015). Presente y futuro de los negocios de barrio (mypes). Universidad del Norte, Escuela de Negocios.

Páramo, D. (2015). La tienda de barrio en Colombia, eje de los negocios barriales. Barranquilla: Escuela de Negocios, Universidad del Norte

Portes, A. y Lauren B. (1987), Desarrollo industrial y absorción laboral: una reinterpretación, Estudios Sociológicos, El Colegio de México, enero-abril, 111-137. https://www.jstor.org/stable/40419915

Rivera-Huerta, R., López, N., y Mendoza, A. (2016). Políticas de apoyo a la productividad de la microempresa informal ¿dónde está México? Problemas del desarrollo, 47(184), 87-109. https://doi.org/10.1016/j.rpd.2016.01.005

Sánchez, M. (2005). Breve inventario de los modelos para la gestión del conocimiento en las organizaciones. Acimed, 13(6), 18. https://bit.ly/3jKTzAA

Telégrafo, E. (25 de julio de 2015). https://www.eltelegrafo.com.ec/noticias/Economia/4/300-MilPersonas-Laboran-En-Las-Tiendas-De-Barrio. El Telégrafo.

Ventura, L. J. (2017). ¿Población o muestra?: Una diferencia necesaria. Revista cubana de salud pública, 43(4), $\quad 0 . \quad$ http://scielo.sld.cu/scielo.php?script=sci_arttext\&pid=S0864$34662017000400014 \& \operatorname{lng}=\mathrm{es} \&$ tlng=es.

Villanueva, J. (2 de septiembre de 2018). Los tenderos organizados en Ecuador tienen una aplicación para conectarse con sus proveedores. El Universo. https://bit.ly/3vZeyEw

Chávez Cruz, G., Campuzano Vásquez, J., y Betancourt Gonzaga, V. (2018). Las Micro, Pequeñas y Medianas Empresas. Clasificación para su estudio en la carrera de Ingeniería en Contabilidad y Auditoría de la Universidad Técnica de Machala. Revista Conrado, 14(65), 247-255. http://conrado.ucf.edu.cu/index.php/conrado 